\title{
Explaining the entropy concept and entropy components
}

\author{
"Theory without practice is empty and practice without theory is blind." \\ Immanuel Kant
}

\section{Marko Popović}

Department of Chemistry and Biochemistry, Brigham Young University, Provo, UT 84602, USA

Email: popovic.pasa@gmail.com

\begin{abstract}
Total entropy of a thermodynamic system consists of two components: thermal entropy due to energy, and residual entropy due to molecular orientation. In this article, a three-step method for explaining entropy is suggested. Step one is to use a classical method to introduce thermal entropy $S_{T M}$ as a function of temperature $T$ and heat capacity at constant pressure $C_{p}: S_{T M}=\int\left(C_{p} / T\right) d T$. Thermal entropy is the entropy due to uncertainty in motion of molecules and vanishes at absolute zero (zero-point energy state). It is also the measure of useless thermal energy that cannot be converted into useful work. The next step is to introduce residual entropy $S_{0}$ as a function of the number of molecules $N$ and the number of distinct orientations available to them in a crystal $m: S_{O}=N k_{B} \ln m$, where $k_{B}$ is the Boltzmann constant. Residual entropy quantifies the uncertainty in molecular orientation. Residual entropy, unlike thermal entropy, is independent of temperature and remains present at absolute zero. The third step is to show that thermal entropy and residual entropy add up to the total entropy of a thermodynamic system $S: S=S_{O}+S_{T M}$. This method of explanation should result in a better comprehension of residual entropy and thermal entropy, as well as of their similarities and differences. The new method was tested in teaching at Faculty of Chemistry University of Belgrade, Serbia. The results of the test show that the new method has a potential to improve the quality of teaching.
\end{abstract}

Keywords: thermal entropy; residual entropy; molecular motion; molecular orientation; education.

\section{Introduction}

"Whoever uses the term 'entropy' in a discussion always wins since no one knows what entropy really is, so in a debate one always has the advantage" are words of Von Neumann that show the widespread misunderstanding of the entropy concept. (Gillet, 2006) Entropy is an abstract concept and is thus harder to explain than other thermodynamic properties, such as energy, pressure and temperature. We encounter energy often in our everyday lives, as calorie charts on our food or kWh on our electricity bills, but we don't have much contact with entropy outside an academic environment. The study of Carson and Watson (2002) shows that there are a lot of misconceptions about entropy among undergraduate students, many of which appeared before they even took a physical chemistry course. Sozbilir and Bennet (2007) tested undergraduate students who took a phys- 
ical chemistry course and found that the majority could not use thermodynamic principles to explain change in entropy of a system. Kostic (2014) found that the concept of entropy is often misused, even in scientific literature. On the other hand, entropy is a key concept in natural sciences, as sir Edington stated: "The law that entropy always increases holds, I think, the supreme position among the laws of Nature..." (Edington, 1928).

While teaching about entropy is not easy, it is even harder to explain its components, such as the residual entropy. Understanding residual entropy requires students to think at a molecular level. This is a problem for many students (Sozbilir \& Bennet, 2007). As a result, there is a general lack of fundamental understanding of residual entropy, even in academic circles.

Residual entropy is easiest to explain as configurational entropy, a concept that students can grasp intuitively. As is shown in section "Entropies considered," residual entropy is easiest to present to students using a coin-toss model. This implies the analysis of residual entropy as configurational entropy (Kozliak, 2007). There has been a debate on whether configurational entropy should be thought to undergraduate students (Kozliak, 2009; Lambert, 2007; Langbeheim et al., 2014). Kozliak (2009) suggests that students should be simultaneously thought about applications and warned about limitations of configurational entropy. Some authors (Lambert, 2007) oppose introducing configurational entropy altogether in introductory courses, as it does not convey the idea that every change in entropy entails a special redistribution of energy. However, these authors (Lambert, 2007) agree that configurational entropy is the only practical method to solve certain problems in thermodynamics, such as finding entropies of mixing (Lambert, 2007). Such systems are encountered by students in everyday phenomena as well as in materials of chemical interest (Lambert, 2007). According to Langbeheim et al. (2014), configurational entropy also plays an important role in shaping conceptual understanding. Research showed that the grasp of configurational entropy is intuitive for students (Langbeheum et al., 2014; Shultz \& Coddington, 1981) and should not be therefore overlooked but rather harnessed, elaborated, and refined (Langbeheum et al., 2014).

Molecules (particles) can be classified into two groups, depending on their symmetry: symmetric particles (Figure 1a) and asymmetric particles (Figure 1b). Symmetric particles can take only one orientation in a crystal and thus form only perfect crystals, where all particles are oriented in the same way. Asymmetric particles can take multiple orientations in a crystal and can form both perfect (Figure 1c) and imperfect crystals (Figure 1d).

a)

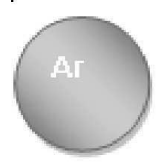

b)

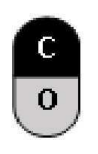

c)

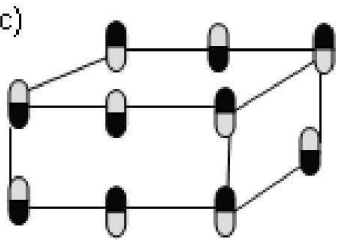

d)

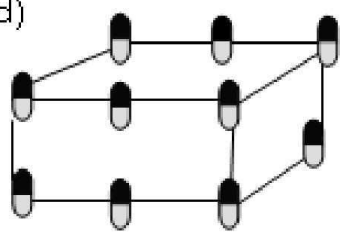

Figure 1. (a) A symmetric particle: in this case an argon atom, which can have only one orientation in a crystal lattice. (b) An asymmetric particle: in this case $\mathrm{CO}$, which can have two orientations in a crystal lattice: either $\mathrm{C}$ or $\mathrm{O}$ can point upwards. (c) An imperfect crystal of $\mathrm{CO}$ : each $\mathrm{CO}$ molecule in the crystal lattice can point either up or down. Therefore, there is randomness and thus uncertainty in molecular orientation, leading to residual entropy. (d) A perfect crystal of CO: all molecules in the lattice are oriented in the same direction. There is no randomness nor uncertainty and, thus, residual entropy is zero. 
In this paper, a new method of explaining entropy to undergraduate students is proposed. The comprehension of residual entropy will be improved if total entropy of a system is explained to them immediately as a sum of its two components. The first is the residual entropy, entropy that is caused by uncertainty in molecular orientation - a static property that survives even in the total absence of motion at absolute zero (zero-point energy state). The second is thermal entropy, entropy that is caused by uncertainty (Boltzmann, 1974) in the motion of particles - a dynamic property that is related to energy (Clausius, 1867; Clausius, 1870; Clausius, 1865). The "Entropies considered" section considers the two components of entropy and how they are related. Section "Teaching strategy" gives the method of explaining them based on the theory in the section "Entropies considered". The "Teaching strategy" section also gives the results of implementation of the new teaching method in the classroom and its comparison by testing to the classical teaching method.

\section{Entropies considered}

There are three terms related to entropy used in thermodynamics: thermal entropy, residual entropy and total system entropy. In this section the three entropies, as well as the relationships, similarities and differences between them will be presented. Based on this a new teaching method will be presented in the next section.

Thermal entropy is a property of a thermodynamic system that results from motion of particles in the system (Boltzmann, 1974). This includes motion such as translation, rotation, vibration, electronic energy, nuclear energy, etc. Thus, thermal entropy is proportional to the amount of energy stored as chaotic molecular motion (Boltzmann, 1974). Thermal entropy $S_{T M}$ at a temperature $T$ is defined and calculated as

$$
S_{T M}=\Delta_{p t} S+\int_{T=0}^{\tau} \frac{C_{p}(T)}{T} d T
$$

$C_{p}$ is heat capacity at constant pressure, $T$ is temperature, and $\Delta_{p t} S$ is the entropy change due to phase transitions.

$\Delta_{p t} S$ can be found from enthalpy of a phase transition $\Delta_{p t} H$ and the temperature of the phase transition $T_{p t}: \Delta_{p t} S=\Delta_{p t} H / T_{p t}$. This equation should theoretically be used for first order phase transitions, because they appear as infinitely tall peaks of zero width (Figure 2a), so the integral of $C_{p} / T$ should be zero. However, in practice, phase transitions do not appear as infinitely narrow peaks and have finite height and width (Figure 2b). Therefore, $\Delta_{p t} S$ is calculated in 4 steps: (1) fit the heat capacity data at temperatures below and above the phase transition. (2) Extrapolate the fitted heat capacity underneath the phase transition peak. (3) Subtract the extrapolated heat capacity from the actual heat capacity data. This gives the heat capacity of the phase transition $C_{p, p t}$ (Figure 2c). (4) Fit and integrate the heat capacity of the phase transition using the equation $\Delta_{p t} S=\int\left(C_{p, p t} / T\right) \mathrm{d} T$. Since this term has the same form as the second term on the right-hand side of equation (1a), we may simplify equation (1a) into

$$
S_{T M}=\int_{T=0}^{\tau} \frac{C_{p}(T)}{T} d T
$$


Since an infinitesimal amount of heat that the system receives is defined as $d Q=C_{p} \cdot d T$, thermal entropy is related to the heat given to the system on heating it from absolute zero to its current temperature $t$. Since this heat was stored as energy of molecular motion within the system, thermal entropy is proportional to it. So, we may assume thermal en-
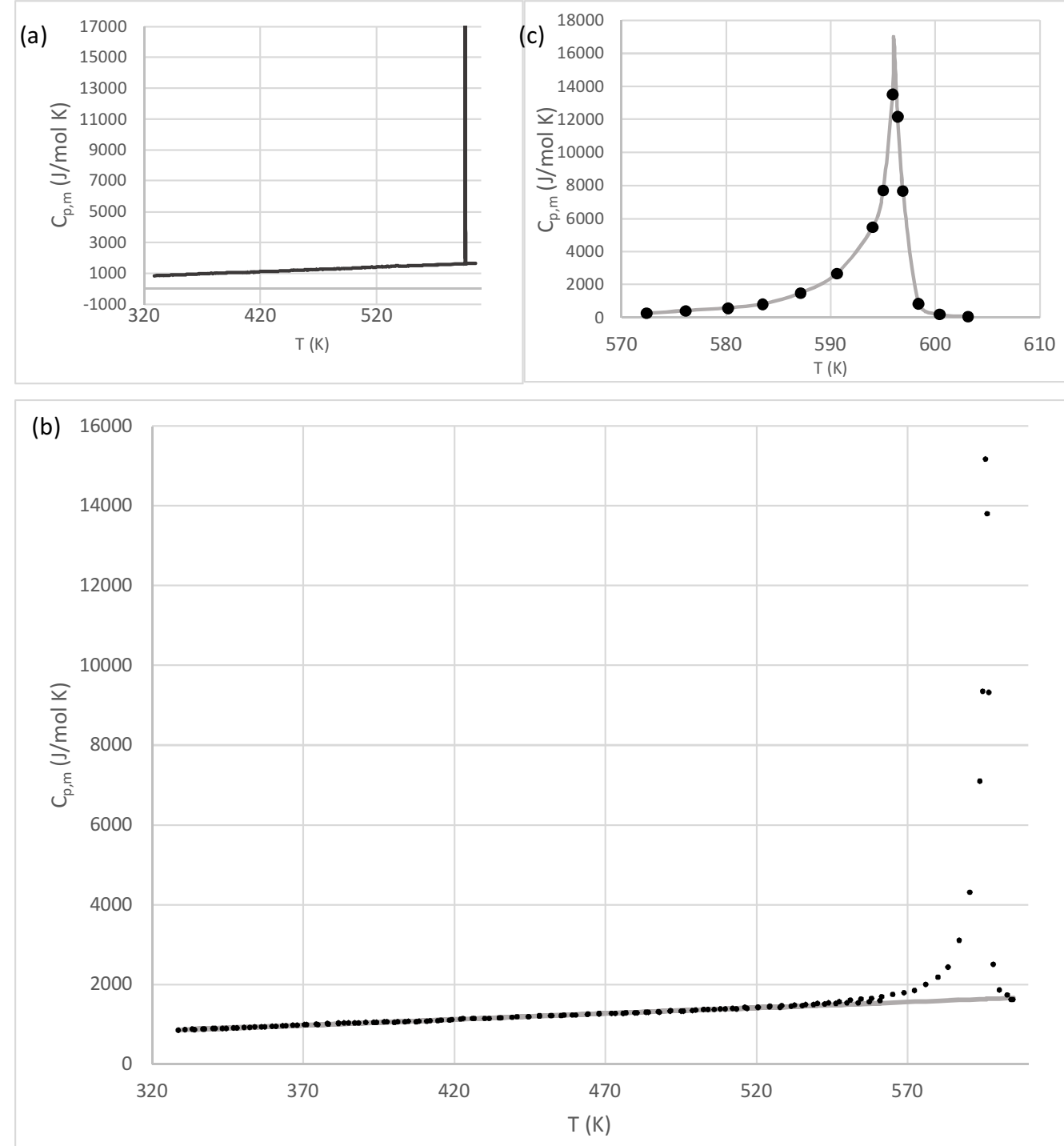

Figure 2. (a) Heat capacity of a hypothetical sample with an ideal first order phase transition. The phase transition peak is infinitely high and infinitely narrow. (b) Heat capacity of a real sample (Smirnova et al., 2005). The points represent experimental data. Notice that the phase transition peak has finite height and width. The line represents fitted heat capacity from the region outside the phase transition. The line segment underneath the phase transition is an extrapolation. (c) Heat capacity of a phase transition is obtained by subtracting the fitted heat capacity line in (b) from the real heat capacity of the sample. The points represent the heat capacity of the phase transition. The line represents a fit made to integrate the phase transition heat capacity. 
tropy to be the entropy that appears as a consequence of molecular motion inside the system. Thus, thermal entropy represents a measure of (various forms of) stored kinetic energy that cannot be converted into useful work at a given temperature (Clausius, 1867; Clausius, 1870; Clausius, 1865).

Residual entropy, $S_{0}$, is a property of a thermodynamic system near (and at) absolute zero that appears as a consequence of random molecular arrangement of asymmetric particles aligned in a crystal lattice (Figure 1b and c) (Clayton \& Giauque, 1932; Johari, 2010; Kozliak, 2007; Kozliak \& Lambert, 2008; Popovic, 2014; Popovic, 2015; Sestak et al., 2011). Kozliak (2007) defined four ways to calculate residual entropy. Informational or combinatoric method, derived using the coin tossing model, is traditionally used in textbooks (Kozliak, 2007). It applies the Boltzmann-Planck formula:

$$
S_{0}=k_{B} \ln \frac{W_{\text {rand }}}{W_{\text {perf }}}
$$

$k_{B}$ is the Boltzmann constant, while $W_{\text {rand }}$ and $W_{\text {perf }}$ correspond to the numbers of microstates of the real (imperfect) and perfect crystals, respectively (Figure 1c and d) (Kozliak, 2007). By definition $W_{\text {perf }}=1$. The quantity $W_{\text {rand }}$ is related to the number of distinct orientations a molecule can have in an imperfect crystal, $m$, and the number of molecules that form the crystal, $N$, by the relation $W_{\text {rand }}=m^{N}$. So, residual entropy is

$$
S_{0}=N \cdot k_{B} \cdot \ln m
$$

According to (3) residual entropy depends only on the number of orientations that molecules can have. So, residual entropy depends only on the number of molecules in a crystal, their arrangement, and is independent of molecular motion - temperature. Therefore, it persists at absolute zero.

Both residual entropy and thermal entropy measure uncertainty. However, they differ in the kind of uncertainty that they quantify: residual entropy is related to uncertainty in arrangement, while thermal entropy is related to uncertainty in energy and motion. By comparing equations (1b) and (3) we can see the main difference between the thermal entropy and the residual entropy. From (3) it can be concluded that residual entropy is related to the number of molecules in the crystal and the number of distinct orientations they can have, $m$. So, residual entropy is caused by uncertainty in molecular orientation and is a static property. From (1b) it follows that thermal entropy depends on temperature and is caused by molecular motion. Thus, thermal entropy is a dynamic property related to energy (Clausius, 1867; Clausius, 1870; Clausius, 1865). At absolute zero all molecules are in their ground state, so there is no uncertainty in their energy. When uncertainty in energy disappears at absolute zero, thermal entropy is equal to zero.

Total system entropy: Entropy is an additive property. Therefore, total system entropy is the sum of two types of entropy: residual entropy and thermal entropy. Total entropy of a thermodynamic system $S$ at a temperature $\tau$ is calculated from the equation

$$
S=S_{0}+\int_{T=0}^{\tau} \frac{C_{p}(T)}{T} d T
$$


where $S_{0}$ is the residual entropy, $T$ is temperature, and $C_{p}(T)$ is heat capacity at constant pressure. The second term on the right side of (4) represents thermal entropy. So, total system entropy is the sum of thermal and residual entropy. Thus, we can write total system entropy as

$$
S=S_{0}+S_{T M}
$$

where $S_{T M}=\int\left(C_{p} / T\right) d T$ and $S_{O}=N k_{B} \ln m$.

This formulation of the total system entropy equation allows us to see the components of the total entropy of a thermodynamic system. Total system entropy is what we find in thermodynamic data tables.

\section{Teaching strategy}

In the previous section the thermal, residual and total system entropy were presented, and their relationships defined. Based on this, in this section a 3-step method for explaining the three kinds of entropy will be presented. The general pattern of explanation follows the order in section 2: start from thermal entropy, then introduce residual entropy and in the end show that they are both constituents of total system entropy. Finally, implementation in the classroom of the new teaching method will be considered, as well as its comparison to the classical teaching method by means of testing.

The first step is to derive thermal entropy equation (1b) the classical way and explain that it depends on energy. One can introduce $d S_{T M}=d Q_{r e V} / T$ straight away or start from the Carnot cycle, derive the Clausius inequality and discuss its meaning. Next, the experimental determination of entropy can be discussed by talking about calorimetry, and how $d Q_{r e v}$ in the entropy definition can be replaced by $C_{p} \cdot d T$ and integrated to yield the expression for thermal entropy (1b). Thermal entropy is a measure of useless thermal energy in the system, which can't be converted into useful work (Clausius, 1867; Clausius, 1870; Clausius, 1865). It is a measure of uncertainty due to energy of the chaotic motion of molecules. However, if one tries to use (1b) to find entropy in practice it will work on most compounds, but there are exceptions, like $\mathrm{CO}$, indicating that something is missing. This sets the stage to talk about the residual entropy.

The next step is to introduce residual entropy and explain its difference from thermal entropy. Explain that orientation is important; $\mathrm{CO}$, water or glycerol can be used as examples. Orientation can be taken into account using the Boltzmann equation. It will be exciting for most students to work with it, because of its fame. You can use elementary combinatorics to show that $W_{\text {rand }}=m^{N}$ and use this to derive the residual entropy equation (3). Then indicate that temperature is absent in (3), while it is the key parameter of the thermal entropy equation (1b). This explains the difference between thermal and residual entropy.

The last step is to show that thermal and residual entropy add up to total system entropy and summarize. Entropy is an additive property and thus thermal and residual entropy add up to the total system entropy. The total system entropy equation is thus (5), followed by ( $1 b)$ and ( 3 ) to make the relationships clearer. Then one can recapitulate, that the total system entropy has two components. Thermal entropy represents a measure of useless energy that cannot be converted into work (Clausius, 1865; Clausius, 1867; Clausius, 1870). It depends on energy and disappears at absolute zero. Residual entropy 
is a measure of uncertainty in arrangement (Clayton \& Giauque, 1932). It depends on arrangement only -it is independent of energy and remains present at absolute zero.

The teaching strategy presented above was tested and compared to the classical strategy by applying both in teaching and then testing the acquired knowledge. The comparison of the test scores revealed that the strategy presented here can potentially give improved teaching results. Two groups of $4^{\text {th }}$ year students were chosen randomly at Faculty of Chemistry, University of Belgrade, Serbia. One group was thought about entropy using the method described in this paper. The other group was thought using the classical method described in most textbooks (Atkins \& de Paula, 2006; Atkins \& de Paula, 2011; Chang, 2000). Next both groups were given an identical test. The test was meant to evaluate three things: understanding of the concept and kinds of entropy, understanding of the physical meaning of entropy, and ability to apply the acquired knowledge. The group that was thought using the method from this paper had an average score of $84 \%$, while the group that was thought according to the classical method had an average score of $63 \%$. Based on the results of the test, it was found that the method presented in this paper has a potential to give students a better understanding and ability to apply the concept of entropy.

\section{Conclusions}

Total entropy of a thermodynamic system has two components: thermal entropy and residual entropy. Thermal entropy is due to uncertainty in motion of molecules. It is also the measure of useless thermal energy that cannot be converted into useful work. Residual entropy quantifies the uncertainty in molecular orientation. Residual entropy, unlike thermal entropy, is independent of temperature and remains present at absolute zero. The three kinds of entropy and their relationships can be explained as follows: start by deriving the thermal entropy equation (1b) in the usual way, mention that except uncertainty in energy there is uncertainty in orientation as well, resulting in residual entropy (3), finally show that thermal entropy and residual entropy add up to the total system entropy (5). The teaching method presented in this paper was tested and compared with the classical teaching method, by means of implementation in the classroom and testing the acquired knowledge. The students thought using the method presented here had an average score of $84 \%$, while the students thought using the classical method had an average score of $63 \%$. This indicates that the method presented in this paper has a potential to give improved results in teaching entropy. Therefore, there are reasons to believe that if the professor uses this method to explain the entropy concept, the students will understand better the concepts of residual entropy, thermal entropy, their similarities and differences.

Acknowledgements: The author would like to express gratitude to prof. Dr. Sanja Grgurić-Šipka, Faculty of Chemistry University of Belgrade without whom the implementation of this teaching approach in classroom would not be possible.

\section{References}

Atkins, P. \& de Paula, J. (2006). Physical chemistry, $8^{\text {th }}$ ed. New York: W. H. Freeman and Company. Atkins, P. \& de Paula, J. (2011). Physical Chemistry for the Life Sciences, $2^{\text {nd }}$ ed. New York: W. H. Freeman and Company. 
Boltzmann, L. (1974). The second law of thermodynamics. In B. F. McGuinness (Ed.), Theoretical physics and philosophical problems (pp. 13- 33). New York: Springer-Verlag.

Carson, E.M. \& Watson, J.R. (2002). Undergraduate students' understandings of entropy and Gibbs free energy, University Chemistry Education, 6, 4-12.

Chang, R. (2000). Physical Chemistry for the Chemical and Biological Sciences, $3^{\text {rd }}$ ed. Sausalito: University Science Books.

Clausius, R. (1867). The Mechanical Theory of Heat - with its Applications to the Steam Engine and to Physical Properties of Bodies. London: John van Voorst.

Clausius, R. (1870). On a Mechanical Theorem Applicable to Heat, Philosophical Magazine, 40(265), 122-127.

Clausius, R. (1865). Ueber verschiedene für die Anwendung bequeme Formen der Hauptgleichungen der mechanischen Wärmetheorie, Annalen der Physik, 201(7), 353-400.

Clayton, J.O. \& Giauque, W.F. (1932). The heat capacity and entropy of carbon monoxide. Heat of vaporization. Vapor pressures of solid and liquid. Free energy to $5000^{\circ} \mathrm{K}$ from spectroscopic data, Journal of the American Chemical Society, 54(7), 2610-2626.

Edington, A.S. (1928). The nature of the physical world. Cambridge: Cambridge University Press.

Gillet, S.L. (2006). Entropy and its misuse, I. Energy, free and otherwise, Ecological Economics, 56(1), 58-70.

Johari, G. (2010). Configurational and residual entropies of nonergodic crystals and the entropy's behavior on glass formation, The Journal of Chemical Physics, 132, 124509.

Kostic, M.M. (2014). The Elusive Nature of Entropy and Its Physical Meaning, Entropy, 16(2), 953967.

Kozliak, E. (2007). Consistent Application of the Boltzmann Distribution to Residual Entropy in Crystal, Journal of Chemical Education, 84(3), 493-498.

Kozliak, E.I. (2009). Overcoming Misconceptions about Configurational Entropy in Condensed Phases, Journal of Chemical Education, 86(9), 1063-1068.

Kozliak, E. \& Lambert, F. (2008). Residual Entropy, the Third Law and Latent Heat, Entropy, 10(3), 274-284.

Lambert, F. L. (2007). Configurational entropy revisited, Journal of Chemical Education, 84(9), 1548-1550.

Langbeheim, E.; Safran, S.A. \& Yerushalmi, E. (2014). Visualizing the Entropy Change of a Thermal Reservoir, Journal of Chemical Education, 91(3), 380-385.

Popovic, M. (2014). Comparative study of entropy and information change in closed and open thermodynamic systems, Thermochimica Acta, 598, 77-81.

Popovic, M. (2015). Are Shannon entropy and Residual entropy synonyms? . In Proceedings of the 2nd Int. Electron. Conf. Entropy Appl., Sciforum Electronic Conference Series, 2, A004. doi:10.3390/ecea-2-A004

Sestak, J.; Mares, J.J. \& Hubik, P. (2011). Glassy, Amorphous and Nano-Crystalline Materials: Thermal Physics, Analysis, Structure and Properties. New York: Springer.

Shultz, T. R. \& Coddigton, M. (1981). Development of the concept of energy conservation and entropy, Journal of Experimental Child Psychology, 31(1), 131-153.

Smirnova, N.N.; Kulagina, T.G.; Markin, A.V.; Shifrina, Z.B. \& Rusanov, A.L. (2005). Thermodynamics of phenylated polyphenylene in the range from $\mathrm{T} \rightarrow 0$ to $640 \mathrm{~K}$ at standard pressure, Thermochimica Acta, 425(1-2), 39-46.

Sozbilir, M. \& Bennet, J.M. (2007). A Study of Turkish Chemistry Undergraduates' Understandings of Entropy, Journal of Chemical Education, 84(7), 1204-1208.

Received: January 31, 2018 Accepted: April 5, 2018 